\title{
On the origin of start-up effects in ply-ply friction for UD fiber-reinforced thermoplastics in melt
}

\author{
Rens Pierik, Wouter Grouve, Sebastiaan Wijskamp and Remko Akkerman \\ Rens Pierik. Faculty of Engineering Technology, Chair of Production Technology, University of Twente, Enschede, The Netherlands \\ ThermoPlastic composites Research Center (TRPC), Enschede, The Netherlands. Corresponding author: Rens Pierik. E-mail \\ address: e.r.pierik@utwente.nl \\ Wouter Grouve. Faculty of Engineering Technology, Chair of Production Technology, University of Twente, Enschede, The \\ Netherlands \\ Sebastiaan Wijskamp. ThermoPlastic composites Research Center (TRPC), Enschede, The Netherlands \\ Remko Akkerman. Faculty of Engineering Technology, Chair of Production Technology, University of Twente, Enschede, The \\ Netherlands \\ ThermoPlastic composites Research Center (TRPC), Enschede, The Netherlands
}

\begin{abstract}
Hot press forming is an attractive production technology to fulfil the increasing demand for complex fiber-reinforced thermoplastic parts. Over the years, process simulation tools on press forming have shown to be very helpful in facilitating the design stage for defect free parts production. One of the important deformation mechanisms considered in process simulations is the relative slip of successive plies or ply-ply friction, of which the underlying principles need to be better understood in order to improve the overall predictive simulation quality. In particular the use of steady-state friction values, neglecting the transient response, is questionable as experiments showed that shear stress overshoots can be as high as three times the long-time value. The phenomenon of the overshoot at start-up shear is analyzed. Possible explanations include nonlinear viscoelasticity and a slip relaxation effect giving rise to wall slip, which are discussed using relevant ply-ply friction measurements carried out on a dedicated friction test set-up. Experimental results on UD C/ PEEK show that the shear stress build up and subsequent relaxation comply with nonlinear viscoelasticity. However, the long-time shear stress fails to match the matrix material's viscosity, possibly due to a yield stress. The flow curve corrected for a yield stress resembles the effects of wall slip. A transient model according to these findings will enhance the accuracy of press forming simulation software.
\end{abstract}

Keywords. Hot Press Forming, Thermoplastic Matrix Composites, Inter-Ply Slip, Ply-Ply Friction, Shear Stress Overshoot

\section{Introduction}

Hot press forming is an attractive processing technology for thermoplastic composites due to the short processing cycles, which allows for cost-effective manufacturing of complex parts with continuous fiber reinforcements [1]. Preconsolidated blanks are heated above melt temperature and subsequently shaped to their desired form by closing carefully designed tooling. The tooling temperature is controlled to regulate the cooling rate and a pressure is applied to obtain a properly consolidated part [2]. The final part quality depends on the tool design and the processing parameters. Engineers rely on dedicated process simulation software to ease tool and process design to enable firsttime-right manufacturing.

Currently, most press-formed parts are relatively simple and are based on woven fabric reinforced blanks with a uniform thickness. However, the industry is moving towards unidirectional (UD) ply-based components with thickness variations and more complex geometries [3]. This shift comes with new challenges, as forming trials on UD plybased laminates showed the occurrence of defects, like fiber wrinkling, that were not present with woven fabrics [4]. More importantly, some of these defects, especially those on a small scale, cannot be predicted with the current simulation approach. Hence, more advanced process simulations tools are needed to enable defect-free-manufacturing 
On the origin of start-up effects in ply-ply friction for UD fiber-reinforced thermopla...

at low costs.

Process simulation for press forming requires a careful mathematical description of the governing deformation mechanisms, most notably intra-ply shear, inter-ply slip and bending [2]. None of these mechanisms is negligible or dominant, as the formability is said to be a result of a 'delicate balance' between them [4]. Improvement of the predictive quality therefore requires, among others, more advanced constitutive modelling of each of the deformation mechanisms. The current study focuses in particular on the inter-ply slip mechanism. Ply-ply slip is essential in curved parts, as the relative movement of subsequent ply layers releases compressive stresses in an inner radius bend and consequently prevents fiber buckling $[5,6]$.

Current simulation approaches often use a steady-state slip coefficient to describe ply-ply friction, neglecting any transient (start-up) effects. However, the initial transient response might be the key to improve the predictive quality. Especially if one considers that the relative displacement of adjacent plies in the forming process is typically small [2, 7]. Hence, the direct objective is to characterize and describe the full nonlinear ply-ply slippage response considerably more accurately for the use in forming simulation software with improved predictive capability for forming defect generation. As a first step, this study focuses on the identification of the underlying governing mechanisms, which will form the basis for future constitutive models.

\section{Hypotheses for the shear stress overshoot}

Ply-ply slip is often characterized by pulling one or multiple plies out of a stack of plies at a constant rate, while recording the resulting pull force, which can be converted into a shear stress [e.g. 2, 7, 8]. A typical ply-ply friction response is depicted in Figure 1a and generally consists of an overshoot, followed by a decay leading towards a steady-state or long-time value [2]. Although the transient response, including an overshoot, has been recognized a long time ago [e.g. 5, 8], a clear view on the origin is still lacking to the best of authors' knowledge. Therefore, two possible explanations for the overshoot will be discussed briefly using the numbered boxes in the schematic ply-ply cross-section shown in Fig. 1b.

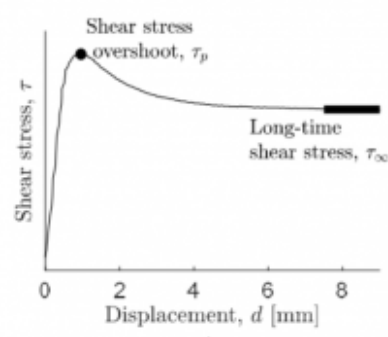

a)

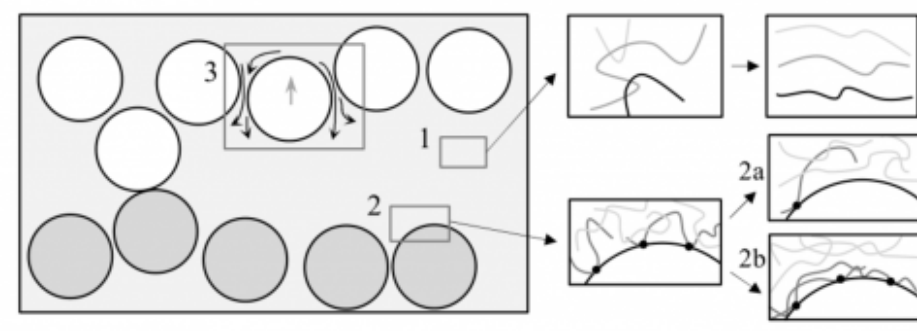

b)

Fig. 1. A typical friction response for ply-ply slip (a) and schematic ply-ply cross-section (b) with polymer melt's response (Box 1) and fiber-polymer interaction (Box 2) as possible mechanisms for the transient response. Resin percolation (Box 3) will be adressed in the discussion.

The first explanation is that the shear stress overshoot is caused by nonlinear viscoelasticity (NLVE) [6]. The transient behavior seen in polymer melts is due to the entangled structure formed by the macromolecules [1,9]. At low shear rates within the linear VE region, the shear stress monotonically increases leading towards a steady-state value. However, at higher deformation rates, the chain diffusion process is not able to accommodate to the deformation fast enough and the junctions act as permanent bonds leading to a rubber-like response. The shear stress increases 
until the chains slip in these junctions, which partly destroys the temporary network of entangled polymer chains (schematically shown in Box 1 in Fig. 1b) [9]. This 'catastrophic realignment' is accompanied by a decrease in the shear stress [1], giving rise to an overshoot.

Alternatively, Sachs [2] proposed a slip relaxation effect as a second explanation for the overshoot in a study on tool-ply friction of UD C/PEEK. Hatzikiriakos and co-workers [10-12] discussed a slip relaxation effect in their studies on wall slip in polymer melts. Two wall slip mechanisms are generally accepted, namely desorption of macromolecules at the wall, or fiber in the present case as shown in Box 2a, or disentangling of adsorbed chains from the bulk (Box 2b) [10, $13,14]$. The relaxation of chains next to the wall differs from those in the bulk, which could result in delayed slip [10]. Hence, a slip relaxation effect might become active, explaining the overshoot observed as a gradual rise in wall slip.

Ply-ply friction experiments have been performed to test the viability of these hypotheses. The materials and methods will be discussed next, followed by a presentation of the results and a discussion.

\section{Materials and methods}

\subsection{Materials and testing set-up}

The material under investigation consists of carbon fibers with a poly(ether-ether-ketone) (PEEK) matrix in the form of unidirectional tape known as Toray Cetex® TC1200, manufactured by Toray Advanced Composites. According to the manufacturer, the melting and processing temperature equal $343{ }^{\circ} \mathrm{C}$ and $385^{\circ} \mathrm{C}$ respectively. The viscosity of the matrix material, Victrex PEEK 150P, was measured using a plate-plate rheometer.

Ply-ply friction tests were performed on a benchmarked friction tester schematically shown in Fig. 2a [2]. A specimen consists of two outer plies and a middle ply with all fibers aligned in the longitudinal direction, as visualized in Fig. $2 \mathrm{~b}$. The specimen was mounted in a universal testing machine. The velocity of the upper clamp can be controlled resulting in a relative movement between the middle ply and the outer plies. The resulting pull force, $\mathrm{F}_{\mathrm{p}}$, was measured using a 1 $\mathrm{kN}$ loadcell, which was used to calculate the shear stress:

$$
\tau=\frac{F_{p}}{2 A}
$$

with $\mathrm{A}$ the area of the heated pressure plates $\left(50 \times 50 \mathrm{~mm}^{2}\right)$. A normal force can be applied on the plates, which was measured using three loadcells. The pressurized area remained constant by using an additional overlap of $15 \mathrm{~mm}$ as shown in Fig. 2a. 


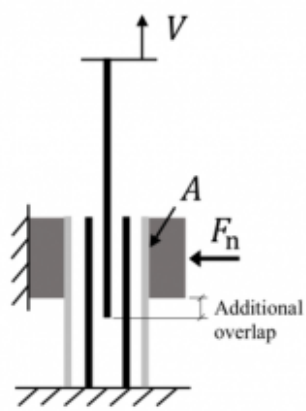

a)

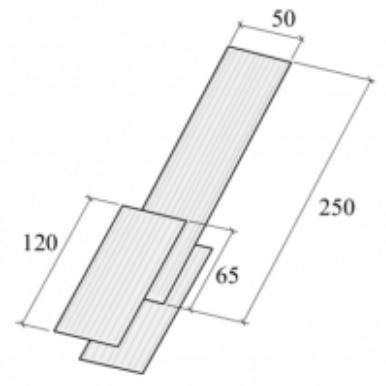

b)

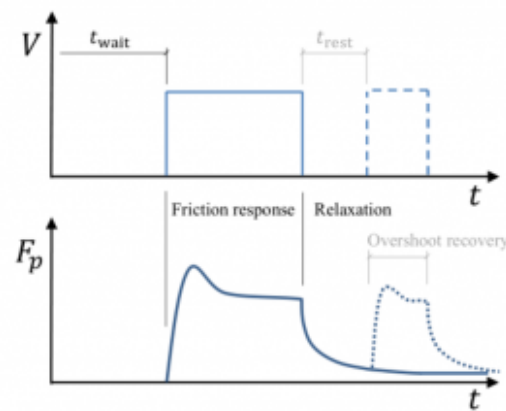

c)

Fig. 2. Overview of the friction tester with a specimen for ply-ply friction (a), schematic drawing of a specimen in mm

(b) and experimental procedure with applied velocity and measured pull force for an ordinary experiment (solid lines) and an interrupted velocity experiment (solid and dashed lines) (c).

\subsection{Test conditions}

Friction experiments were performed to obtain insight in the transient friction response and to investigate the origin of the shear stress overshoot. Fresh specimens were used for each experiment. The temperature and pressure were held constant, reading $385{ }^{\circ} \mathrm{C}$ and $15 \mathrm{kPa}$, respectively, whereas the sliding velocities ranged from 1 to $200 \mathrm{~mm} / \mathrm{min}$. A waiting time of five minutes was used to let the matrix melt and to heal the adjacent plies before testing. The solid lines in Fig. 2c represent the applied velocity and resulting pull force with time during an ordinary sliding experiment. The transient friction response is of interest, but comparison of the steady-state values with the matrix material's viscosity is interesting as well to evaluate the hypotheses presented earlier. Further, subsequent relaxation after cessation of shear deformation could indicate NLVE. Additionally, the sliding action was interrupted in some experiments for a certain rest time to analyze the recovery of the overshoot, as shown by the solid and dashed lines in Fig. 2c. The recovery, and especially the timescales involved, could distinct NLVE from another mechanism. These experiments were conducted at a rate of $25 \mathrm{~mm} / \mathrm{min}$.

\section{Results}

\subsection{Ply-ply friction response}

The ply-ply friction response of UD C/PEEK is shown in Figure 3a for different rates. Several measurements at $25 \mathrm{~mm} /$ min, plotted in gray, indicate a fair repeatability of the measurements. The response at low rate follows a monotonic stress growth, whereas higher rates cause a shear stress overshoot, $\tau_{p}$, leading towards a long-time shear stress, $\tau_{\infty}$.

Stress relaxation occurs when the test is stopped. Normalized relaxation curves are shown in Fig. 3b, which are corrected for the residual stress after full relaxation, around $2 \mathrm{kPa}$ for each experiment, and plotted on logarithmic scale with time to emphasize the difference in relaxation rate with sliding velocity. The second friction response in the interrupted velocity experiments, in which the sliding velocity was restarted after different rest times, is shown in Fig. 3c. The overshoot is almost fully recovered after more than $30 \mathrm{~s}$, whereas a small overshoot is already visible after $1.2 \mathrm{~s}$. 


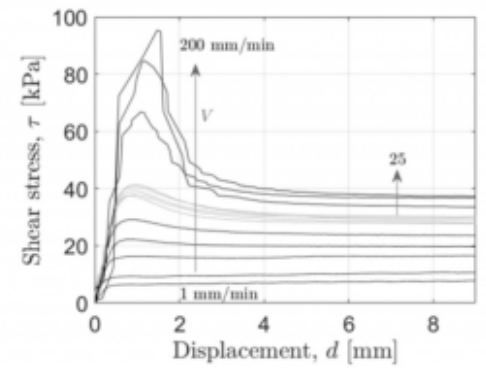

a)

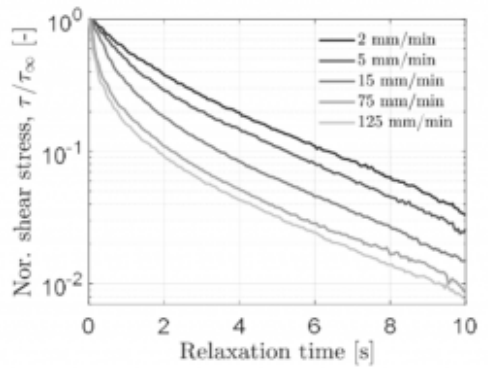

b)

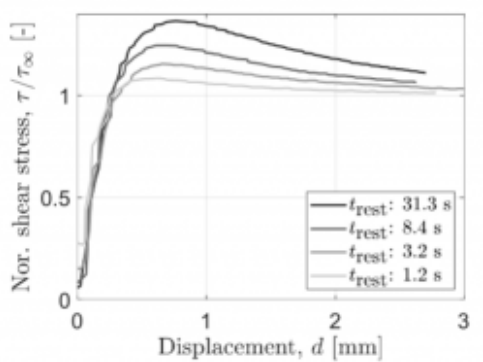

c)

Fig. 3. Ply-ply friction response of UD C/PEEK at $385^{\circ} \mathrm{C}$ and $15 \mathrm{kPa}$ under influence of sliding velocity with shear stress vs. displacement (a) and subsequent normalized relaxation curves (b). Effect of rest time on the recovery of the overshoot at $25 \mathrm{~mm} / \mathrm{min}$ (c).

\subsection{Shear stress overshoot}

A closer look at the stress build up is shown in Fig. 4a. The monotonic shear stress increase at low velocities changes into a curve exhibiting an overshoot, which reduces in width and increases in magnitude with sliding velocity. The ratio of $\tau_{\mathrm{p}}$ and $\tau_{\infty}$ is plotted in Fig. $4 \mathrm{~b}$ and indicates that the overshoot starts to occur at velocities higher than $0.1 \mathrm{~mm} / \mathrm{s}$ (or $6 \mathrm{~mm} / \mathrm{min}$ ). The ratio rapidly increases up to almost a factor of three.

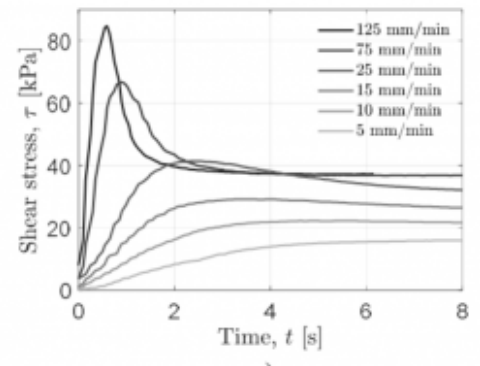

a)

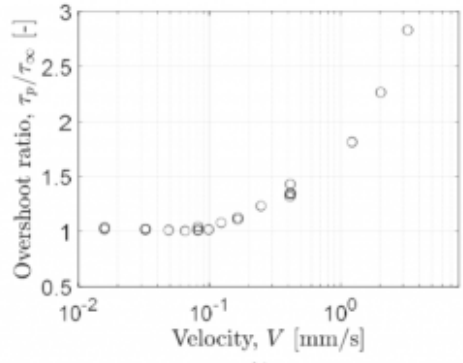

b)

Fig. 4. Shear stress growth with time for several rates (a) and ratio of overshoot and its long-time shear stress with velocity (b).

\subsection{Long-time shear stress}

The long-time shear stress, $\tau_{\infty}$, is evaluated as the mean in the steady region, as indicated in Figure $1 \mathrm{a}$. A relation between $\tau_{\infty}$ and the matrix material's viscosity is expected if a thin resin-rich layer is present at the slip interface. The viscosity of PEEK 150P, as measured by plate-plate rheometry, is plotted in Fig. 5 a for 380 and $390{ }^{\circ} \mathrm{C}$. The Cross model [15], 
On the origin of start-up effects in ply-ply friction for UD fiber-reinforced thermopla...

$$
\eta_{\mathrm{c}}(\dot{\gamma})=\frac{\eta_{0}}{1+\left(\frac{\eta_{0} \dot{\gamma}}{\tau^{*}}\right)^{1-n}}
$$

with $\eta_{0}$ the zero shear rate viscosity, $\tau^{*}$ the critical shear stress at the onset of shear thinning and $\mathrm{n}$ the Power Law Index, was used to fit the viscosity with shear rate, $\dot{\gamma}$, on $385^{\circ} \mathrm{C}$ by means of interpolation. Equation 2 can be rewritten as,

$$
\tau_{\mathrm{C}}(V, h)=\frac{\eta_{0}}{1+\left(\frac{\eta_{0}(V / h)}{\tau^{*}}\right)^{1-n}} \frac{V}{h}
$$

with $\mathrm{V}$ the sliding velocity and $\mathrm{h}$ the film thickness to compare the viscosity and friction data in terms of shear stress. A straightforward relation between $\tau_{c}$ and $\tau_{\infty}$ could not be drawn using a constant film thickness, as visualized in Fig. $5 b$. However, a correction can be applied to $\tau_{\infty}$ by subtracting the residual stress after full relaxation, $\tau_{y}$, possibly attributed to fiber-fiber contact. The result is shown in Fig. $5 c$ together with $\tau_{c}$ using a film thickness of $2.5 \mu \mathrm{m}$.

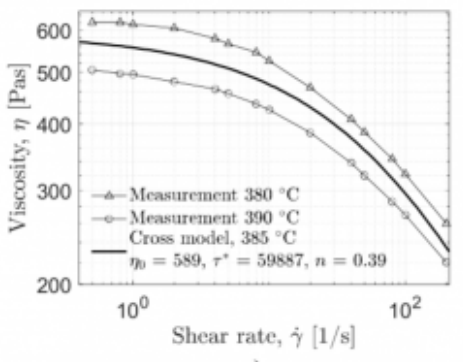

a)

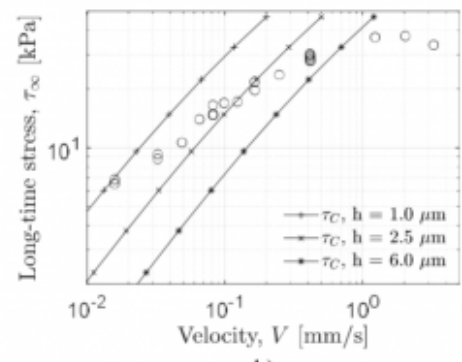

b)

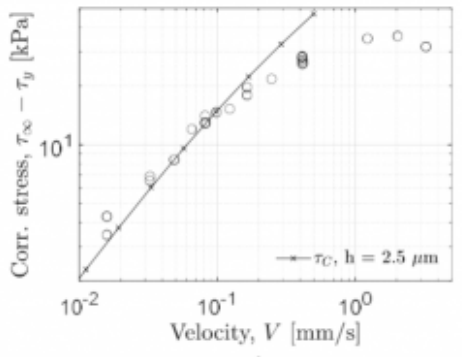

c)

Fig. 5. Viscosity of Victrex PEEK 150P with a Cross model fit at $385^{\circ} \mathrm{C}$ (a), which is compared with $\tau_{\infty}$ from the friction tests by using several film thicknesses (b) and corrected $\tau_{\infty}$ for the residual stress after full relaxation, $\tau_{c}$, shown with the Cross model fit using a film thickness of $2.5 \mu \mathrm{m}$ (c).

\section{Discussion}

The experimental observations will be used to evaluate the hypotheses, namely nonlinear viscoelasticity (NLVE) and a slip relaxation effect giving rise to wall slip, to shed light on the mechanism responsible for the shear stress overshoot.

Nonlinear viscoelasticity complies with several experimental observations. The monotonic stress increase at low rates, while an overshoot appears at higher rates that shifts towards shorter times with a more narrow and larger peak (Fig. 4a), shows a striking similarity with NLVE [9]. The relaxation curves, shown in Fig. 3b, are straight lines at low rate resembling linear $\mathrm{VE}$, whereas the relaxation rate increases at higher velocities. The higher relaxation rate could be due to the new structure formed by the oriented chains with less entanglements $[1,9]$. All curves tend to become parallel with time, as the original structure recovers during relaxation. Lastly, the interrupted velocity experiments, as illustrated in Fig. 3c, showed that the recovery time for the overshoot is a bit longer than the time required for relaxation, which can be expected since many segmental motions of the macromolecule are required to recover the 
fully entangled network $[15,16]$.

The above suggests NLVE as the mechanism causing the transient friction response. However, the shear strain unit at the overshoot peak conflicts with the notion of Schweizer [17], who stated that this value should be around 2 at the first emergence of an overshoot based on experiments at different laboratories on several polymer systems. When looking at the $\mathrm{C} / \mathrm{PEEK}$ data, the shear strain at the overshoot exceeds 250 when using a film thickness of $3 \mu \mathrm{m}$. Further, a straightforward relation between $\tau_{\infty}$ and the matrix material's viscosity could not be established (Fig. $5 \mathrm{~b}$ ), which would be expected in case of NLVE.

A gradual increase of wall slip, or a slip relaxation effect, was also mentioned in section 2, for which the long-time shear stress with velocity as plotted in Fig. $5 \mathrm{c}$ is useful. The correction on $\tau_{\infty}$ by the residual stress after full relaxation, $\tau_{y}$, can be interpreted as a yield stress according to the findings of Murtagh [8] on stress-controlled experiments with UD C/PEEK, possibly due to fiber-fiber contact [1]. A fair correspondence between $\tau_{\infty}-\tau_{y}$ and the matrix material's viscosity using a constant film thickness is obtained at low rates. A deviation with the viscosity curve starts around 0.1 $\mathrm{mm} / \mathrm{s}$; the velocity at which an overshoot emerges. A second kink unfolds around $35 \mathrm{kPa}$, resulting in a stress plateau with even a decay at the highest velocity measured. The observed behavior corresponds to the description of wall slip given by Hatzikiriakos [10], who explained that wall slip starts with flow induced adhesive failure and the number of desorbed chains increases with shear rate. The wall slip regime changes to a sudden disentanglement of adsorbed and bulk chains at a second critical shear stress, which is accompanied by a jump in the flow curve and possibly a decreasing shear stress [10].

As an alternative or addition to the discussed hypotheses, $\tau_{\infty}$ could be influenced by an increasing film thickness with rate, as suggested in Fig. 5b. The film thickness increase could be due to resin percolation towards the slip interface, possibly encouraged by reordering of the fibers due to the pull force, which could cause the observed transient response (see Box 3 of Fig. 1b). However, the concept of wall slip better explains the small width of the overshoot with time at high rates, showing signs of failure (Fig. 4a). A wider overshoot would be expected at higher rates in case of an increasing thickness, as more resin needs to percolate.

\section{Conclusions}

Ply-ply friction tests were performed on unidirectional C/PEEK tape to investigate the nature of the transient response, which exhibits a shear stress overshoot. Hypotheses for this overshoot were outlined, namely nonlinear viscoelasticity (NLVE) and a slip relaxation effect giving rise to wall slip. NLVE seems to comply with most of the experimental observations, but the long-time values do not correspond well with the matrix viscosity. This deviation can be solved for the full velocity range by means of a changing film thickness or at low velocities by introducing a yield stress. However, the overshoot shows signs of failure at higher rates rather than a smooth transition from peak towards steady-state, which would be expected in case of film thickness increase by resin percolation. Furthermore, the flow curve corrected for the yield stress matches with the concept of wall slip. Although wall slip seems to be related to the viscoelasticity of the matrix material, more effort is required to further investigate the relation between the slip relaxation effect and the apparent NLVE characteristics of the friction response. The improved understanding will form the basis for more advanced constitutive ply-ply friction models, leading to improved simulation accuracy, in turn facilitating defect-freemanufacturing using hot press forming.

\section{Acknowledgements}

This work was performed as part of the MaterialenNL research program under project number 17880, which is (partly) financed by the Dutch Research Council (NWO). The authors also gratefully acknowledge the financial and technical support from the industrial and academic members of the ThermoPlastic composites Research Center (TPRC), as 
On the origin of start-up effects in ply-ply friction for UD fiber-reinforced thermopla...

well as the support funding from the Province of Overijssel for improving the regional knowledge position within the Technology Base Twente initiative. Further, the authors would like to thank Marten van der Werff for carrying out the rheometer measurements.

\section{Bibliography}

[1] Advani, S.G. Creasy, T.S. Shuler, S.F. Chapter 8 rheology of long fiber-reinforced composites in sheet forming. In: Bhattacharyya D, editor. Composite materials series. Elsevier, 1997, p. 323-69.

[2] Sachs, U. Friction and bending in thermoplastic composites forming processes. Ph.D. thesis, 2014, University of Twente, Enschede, The Netherlands.

[3] Composites World. Thermoplastics in aerospace composites outlook, 2014-2023, 2014, https://www.compositesworld.com/articles/the-outlook-for-thermoplastics- in-aerospace-composites-2014-2023 12.3.2020

[4] Haanappel, S.P. Forming of UD fibre reinforced thermoplastics. Ph.D. thesis, 2013, University of Twente, Enschede, The Netherlands.

[5] Morris, S.R. and Sun, C.T. An investigation of interply slip behaviour in AS4/PEEK at forming temperatures. Compos. Manuf., 1994, 5 (4), 217-224.

[6] Vanclooster, K. Forming of multilayered fabric reinforced thermoplastic composites. Ph.D. thesis, 2009, KU Leuven, Leuven, Belgium.

[7] Dörr, D. Faisst, M. Joppich, T. Poppe, C. Henning, F. Kärger, L. Modelling approach for anisotropic inter-ply slippage in finite element forming simulation of thermoplastic UD-tapes. AIP Conference Proceedings, 2018, 020005. DOI 10.1063/ 1.5034806.

[8] Murtagh, A.M. Monaghan, M.R. Mallon, P.J. Investigation of the interply slip process in continous fibre thermoplastic composites. Proceedings of ICCM-9, Miravete A, editor, Madrid, 1994, 311-318.

[9] Malkin, A. Ya. Isayev, A. I. Rheology: concepts, methods, and applications. 3 rd ed. ChemTec Publishing, 2017, Toronto, Canada. ISBN: 978-1-927885-22-2. [10] Hatzikiriakos, S.G. Wall slip of molten polymers. Prog. Polym. Sci., 2012, 37 (4), 624-643.

[11] Hatzikiriakos, S.G. Dealy, J.M. Wall slip of molten high density polyethylene. I. Sliding plate rheometer studies. J. Rheol., 1991, 35 (4), 497-532.

[12] Kazatchkov, I.B. Hatzikiriakos, S.G. Relaxation effects of slip in shear flow of linear molten polymers. Rheol. Acta, $2010,49,267-274$.

[13] Ten Thije, R.H.W. Akkerman, R. Ubbink, M. Van der Meer, L. A lubrication approach to friction in thermoplastic composites forming processes. Compos. Appl. Sci. Manuf., 2011, 42 (8), 950-960.

[14] Wang, S.Q. Nonlinear polymer rheology: macroscopic phenomenology and molecular foundation. John Wiley \& Sons, 2017, Hoboken (NJ), USA. ISBN: 978-1- 119-02904-5.

[15] Osswald, T. Rudolph, N. Polymer rheology. Carl Hanser Verlag, 2015, München, Germany. ISBN: 978-1-56990-532-4.

[16] Dealy, J.M. Tsang, WM. K.-W. Structural time dependency in the rheological behavior of molten polymers. J. Appl. 
Polym. Sci., 1981, 26 (4), 1149-1158.

[17] Schweizer, K.S. Xie, S.-J. Physics of the stress overshoot and chain stretch dynamics of entangled polymer liquids under continuous startup nonlinear shear. ACS Macro Lett., 2018, 7, 218-222.

PDF automatically generated on 2021-05-23 07:14:14

Article url: https://popups.uliege.be/esaform21/index.php?id=3695

published by ULiège Library in Open Access under the terms and conditions of the CC-BY License (https://creativecommons.org/licenses/by/4.0) 